\title{
UNIVERSITYOF
}

FORWARD

THINKING

WESTMINSTER用

WestminsterResearch

http://www.westminster.ac.uk/westminsterresearch

Les variétés régionales non-méridionales de France: Nivellement; dédialectalisation; supralocalisation

Pooley, T. and Kasstan, J.

This is a copy of the final version of an article published in Sociolinguistica, 30 (1), pp. 175-198. It is available from the publisher at:

https://dx.doi.org/10.1515/soci-2016-0010

The WestminsterResearch online digital archive at the University of Westminster aims to make the research output of the University available to a wider audience. Copyright and Moral Rights remain with the authors and/or copyright owners.

Whilst further distribution of specific materials from within this archive is forbidden, you may freely distribute the URL of WestminsterResearch: ((http://westminsterresearch.wmin.ac.uk/).

In case of abuse or copyright appearing without permission e-mail repository@westminster.ac.uk 


\section{Les variétés régionales non-méridionales de France: nivellement; dédialectalisation; supralocalisation}

\section{Introduction}

Depuis au moins les dernières décennies du $19^{\mathrm{e}}$ siècle, de nombreux dialectologues (par ex., Dauzat 1906, Brun 1946) ont commenté l'importance évidente du contact entre le français et les langues vernaculaires locales (rurale dans la majorité, mais certainement pas dans la totalité, des cas) dans l'émergence des français régionaux. Mais ces dialectologues étaient en même temps conscients de la variation au niveau infra-régional, même si le modèle théorique dont ils disposaient était insuffisant pour tenir compte des divergences diastratiques qui sous-tendaient la variation documenteé dans l'Atlas linguistique de la France (ALF) (Gilliéron et Edmont 19021910). Il est même remarqué dans la notice à l'ALF que les variétés locales pratiquées dans les petites villes étaient en général plus francisées que celles qu'on entendait dans les villages et que les pratiques les moins francisées s'entendaient dans les hameaux. La contrepartie d'une telle distribution est que la pénétration du français était plus avancée dans les zones urbaines, et Dauzat (1906: 203) y ajoute parmi les couches supérieures de la population: «le français s'est d'abord implanté dans les centres urbains et dans les classes riches: modifié sous l'influence du milieu, il constitue ce que j'appellerai le français régional». La notion de «français régional» couvre donc une large gamme de pratiques, allant des français urbains plus ou moins de bon aloi aux interlangues des bilingues tardifs, et de variation sur les plans géographique, social et stylistique.

Même si le bilinguisme a progressivement diminué au fil des générations dans de nombreuses régions (e.g. Potte 1977, Auzanneau 1998, Léonard 2002, Pooley 2004, Kasstan 2015), la rupture provoquée par les deux conflits mondiaux a donné lieu à une chute marquée du taux de transmission des langues autochtones. La génération née après la deuxième guerre mondiale fait aussi figure, comme nous le montrerons, de génération-charnière pour le recul des variantes régionales, même si on avait encore l'impression pendant quelques décennies que ces formes maintenaient une robuste vitalité: «Ce qu'il faut reconnaître, c'est que les différences sur le plan géographique l'emportent pour le moment, dans nos régions, sur les différences sociales» (Walter, 1982: 52). Cette affirmation basée sur des données recueillies auprès de témoins nés entre 1887 et 1956, a été remise en question à peine quinze ans plus tard par Gadet (1996: 67), qui a signalé aussi l'importance du «primat du diaphasique». 
Les premiers travaux variationistes consacrés à cette phase de saillance diaphasique dans d'autres pays (e.g. Labov 1966, Trudgill 1974) avaient déjà démontré que dans les sociétés occidentales, les accents les plus localisés (et donc localisables du point de vue de la perception) s'entendent parmi les strates moins favorisés de la population, et qu'il est, par contre, bien plus difficile de déceler les origines régionales des couches supérieures. Cette approche de dialectologie urbaine avait produit aussi des résultats impressionnants pour les français du Canada (e.g. Mougeon 1996) en grande partie avant la publication des travaux sur les villes françaises les mieux documentées dans cette perspective: Paris (Laks 1977; 1983, Hansen 1998, Hansen \& Juillard 2011, Jamin 2005); le Nord-Pas-de-Calais - Lille (Lefebvre 1991, Pooley 1996; 2004) et Lens (Hornsby 2006); Aix-Marseille (Taylor 1996, Binisti \& Gasquet-Cyrus 2003, Gasquet-Cyrus 2004; 2009). Mais dans les régions oïliques et francoprovençales (les régions non-méridionales), le primat du diaphasique correspond de plus en plus à l'absence de variabilité diatopique (Armstrong 2001), largement confirmée par des études de perception (par ex., Armstrong et Boughton 1998; 2009).

Le but dans le présent article serait d'analyser cette «homogeneisation inexorable» (Jones \& Hornsby 2013) en nous appuyant sur des exemples de variation relevés dans les zones-urbaines. Des comparaisons inter-générations, comme nous le verrons, permettent de constater le recul de nombreux traits régionaux, mais aussi la quasi-disparition (nivellement) des marques d'origine dialectale. Mais dédialectalisation ne signifie pas pour autant dérégionalisation, car les marques régionales peuvent être perçues différemment d'une génération à l'autre, ou d'une région à l'autre, et aussi se maintenir ou être délaissées pour des raisons différentes. Et l'effacement des différences sur le plan phonologique n'implique nullement que la diversité lexicale est réduite de la même manière : certaines études récentes ont démontré que des traits supralocaux peuvent être porteurs d'identités plus localisées (par ex., Eckert 2000, Pooley 2009, Moore 2010).

Nous allons maintenant présenter les notions clés dans la Section 2, qui sera suivie d'un aperçu de la carte des variétés régionales qu'on peut tracer à partir des études de perception (Section 3), corroborée dans la Section 4 par des études de comportement. Ces études se focalisent sur les locuteurs nés depuis 1970, alors que la Section 5 présente des exemples de nouvelles formes régionales (les français des banlieues, l'appropriation locale de variantes plus largement répandues et les phénomènes en quelque sorte «inaudibles», mais décelables soit par des comparaisons inter-régionales soit par des analyses instrumentales. 


\section{Dédialectalisation, nivellement, supralocalisation}

\subsection{La dédialectalisation}

Si le sens du terme «dédialectalisation» semble clair (c'est-à-dire, que les locuteurs, dont le parler normal maintient des traits caractéristiques de leurs origines géographiques, ont néanmoins tendance à éviter des formes perçues comme dialectales) son emploi est quelque peu problématique, car le statut de ces variétés traditionnellement stigmatisées a été réhaussé au cours des dernières décennies. Il est devenu usuel de considérer commes des «langues» ces variétés appelées historiquement «patois» ou «dialectes» (Blanchet \& Armstrong 2006). En France, l'inventaire de ces langues s'est accru considérablement depuis la Loi Deixonne de 1951, passant de quatre en 1951 à 75 en 1999 et inclut neuf langues d'Oïl et le francoprovençal (Rapport Cerquiglini de 1999). Cette reconnaissance limitée peut être considérée comme une forme de dédialectalisation grâce à la valorisation des formes «authentiques», souvent différenciées des pratiques réelles souvent perçues de façon négative.

Il est également crucial de différencier dédialectalisation et dérégionalisation, car si les locuteurs évitent les variantes stéréotypées, d'autres variantes ont été perçues différemment au fil des générations. Par exemple, l'assourdissement des consonnes finales (e.g. [saf] sage) est employée par les générations lilloises nées dans la première moitié du $20^{\mathrm{e}}$ siècle, et constitue une forme minoritaire pour la génération des baby-boomers de la classe ouvrière (Pooley 1996; 2004). Les générations nées après 1980 de niveau social équivalent l'emploient de façon marginale et certains d'entre eux considèrent que ce trait n'est même pas français, malgré le maintien de sa vitalité parmi toutes les couches sociales de l'autre côté de la frontière belge à Tournai (Hambye 2005).

De plus, la perte des traits dialectaux ne suit pas forcément une logique structurale. Par exemple, les paradigmes morphologiques ne se désagrègent pas de façon uniforme : e.g. les formes de la troisième personne du singulier de l'imparfait des verbes être et avoir - étot et avot - en picard s'emploient plus fréquemment que les formes pluriel étottent et avottent. D’ailleurs, les formes pluriel manifestaient plus de vitalité dans les années 1980 à Avion (Hornsby 2006) qu'à Roubaix (Pooley 1996). Comme la fragmentation structurale ne va pas de pair avec la stigmatisation ou le prestige ouvert et latent, il arrive parfois qu'une forme d'un paradigme se maintienne, alors que d'autres sont délaissées. Parmi les pronoms sujets picard utilisés à Avion c'est alle, qui est la forme la plus utilisée dans les séquences considérées par Hornsby (2006) comme picardes. Mais cette forme largement répandue dans les variétés d'Oïl répertoriées dans l'ALF, figure dans les descriptions du français populaire parisien (e.g. Bauche 1946), ce qui laisse supposer une mesure de prestige latent non-négligeable dans la perception des locuteurs des classes populaires nés au cours des premières décennies du $20^{\mathrm{e}}$ siècle. 


\subsection{Le nivellement et supralocalisation}

Le nivellement linguistique est défini par Williams \& Kerswill (1999: 149) comme suit:

[...] a process whereby differences between regional varieties are reduced, features which make varieties distinctive disappear, and new features emerge and are adopted by speakers over a wide geographical area.

D’autres chercheurs (e.g. Watt 2002) croient qu'il y a deux processus conceptuellement indépendants: la koinéisation - les koinés étant des variétés qui émergent des situations de contact - et le Dialektausgleich ou l'extension de l'aire où une certaine variété se pratique aux dépens de variétés concurrentes. Il semblerait que les deux cas de figure supposent un centre directeur, qui sert de creuset et exerce en même temps une influence socio-culturelle. L'indiscutable domination politique, culturelle et économique de Paris permet à la capitale française de remplir ces deux rôles, et en fait un cas exemplaire du modèle «Centre-Périphérie» (Reynaud 1981). L'influence de Paris se fait sentir à la fois au niveau de la variété de référence (la norme de l'élite) et à celui des variétés vernaculaires qui se propageaient grâce aux migrations de personnes modestes depuis plusieurs siècles (Lodge 2004). Si l'ère industrielle a permis le développement de vernaculaires urbains, la désindustrialisation du dernier quart du 20e siècle a vu une croissance marquée de la périurbanisation, phénomène fortement défavorable au maintien des traits linguistiques localisables, car il contribue à l'urbanisation des campagnes, favorisant un mode de vie composite (Pedersen 1994) où les déplacements se multiplient, et donc propice au Dialektausgleich. Même les vieux quartiers des centre-villes où les pratiques localisées persistent accueillent de nombreux étrangers, pour qui le français est une langue seconde ou parfois subissent un processus de gentrification (par ex., Trimaille et Gasquet-Cyrus 2013). Les dernières générations sont beaucoup plus exposées à une langue sinon standardisée, tout au moins neutre sur le plan diatopique grâce à l'allongement de la scolarisation, à l'expansion du secteur des services et à l'explosion des média audio-visuels. Par conséquent, il est devenu de plus en plus difficile de déceler les origines géographiques des locuteurs des régions nonméridionales. Ce français homogénisé pratiqué par une bonne partie de la population dans plusieurs régions de France peut être qualifié de «supralocal» (Armstrong et Pooley 2010), mais il est loin d'être limité à ces aires dialectales traditionnelles. 


\section{La géographie de l'Imaginaire}

\subsection{La supralocalisation des perceptions}

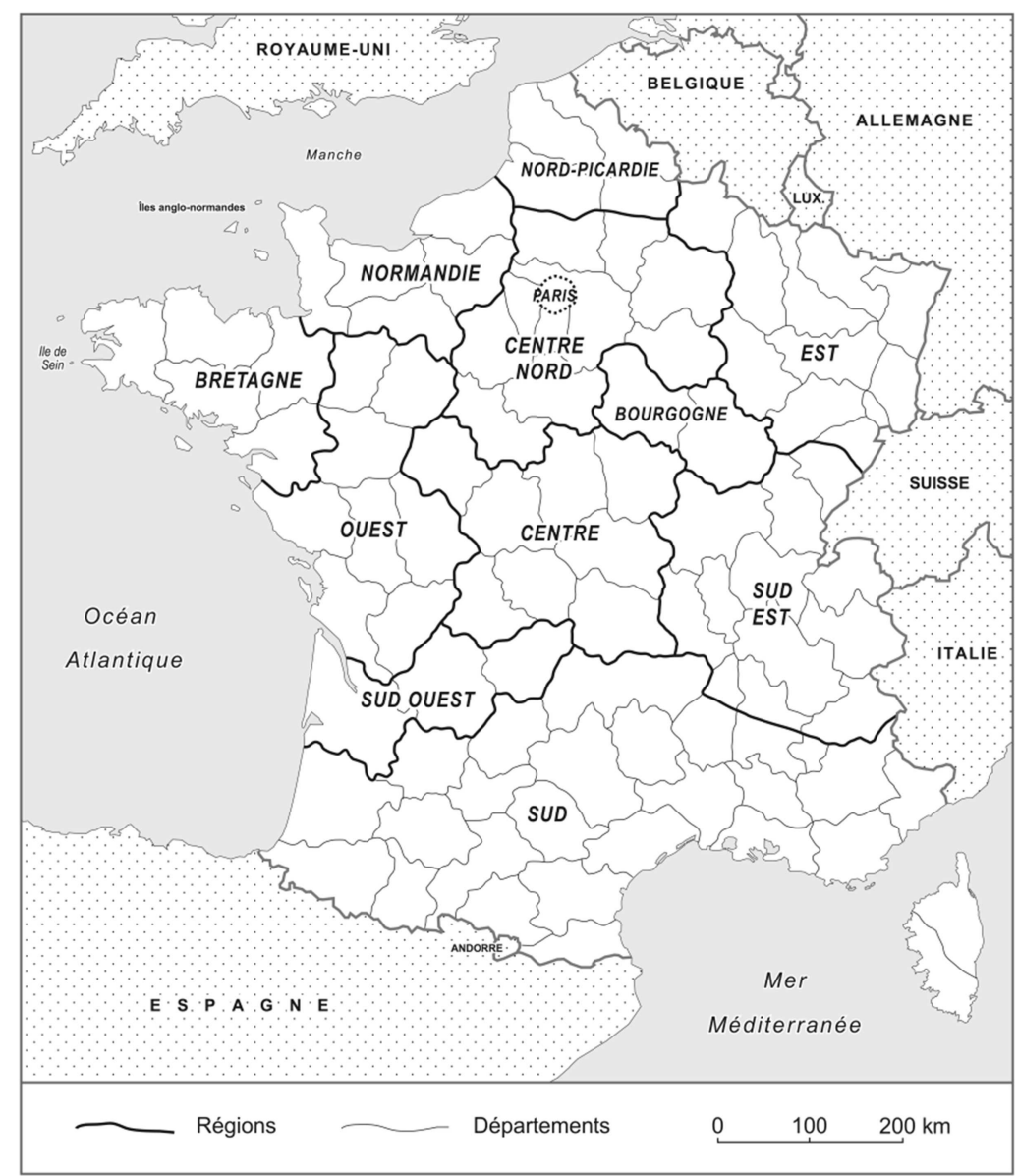

Figure 1: Répartition géographique des accents des hommes des classes moyennes en 1941 (Martinet, 1945)

Déjà dans les années 1940, la première étude sociolinguistique consacrée à la variation régionale (Martinet 1945) montre des indices de supralocalisation. Les témoins, officiers, prisonniers de guerre (donc des hommes des classes moyennes et supérieures et nés entre 1880 et 1920), ont été répartis suivant leur région d'origine (Figure 1). On remarque peu de différences entre les sujets parisiens et ceux qui étaient originaires du centre nord. Les témoins mobiles qui avaient changé plus d'une fois 
de région au cours de leur jeunesse signalaient eux aussi peu de différences avec les deux premiers groupes dans la perception de leur prononciation. Les sujets bretons et alsaciens ne se distinguaient pas de leurs camarades originaires des régions oîliques contigues. Et si on faisait abstraction des allongements vocaliques, les différences entre les diverses parties du domaine d'Oïl seraient bien moins nombreuses.

Des études plus récentes (Armstrong \& Boughton 1998; 2009, Boughton 2005; 2006) montrent que les sujets ordinaires n’arrivent pas à situer des locuteurs au sein de l'aire oïlique, ce qui constitue la majeure partie de l'Europe francophone en termes de surface et de population. Dans leurs enquêtes, Armstrong et Boughton ont demandé à des Rennais d'identifier la région d'origine de locuteurs nancéiens et vice versa sur la base d'un enregistrement assez long (une minute environ). Même les meilleures réponses (peu nombreuses) étaient plutôt vagues (cf. Woehrling \& Boula de Mareüil 2006). Par contre, d'autres études indiquent que les locuteurs sont persuadés que les habitants de leur ville, par ex., le Havre (Hauchecorne et Ball 1997), parlent avec un accent reconnaissable et caractéristique de la localité en question, même si une analyse linguistique ne permet pas de différencier leur façon de parler d'un français vernaculaire assez largement répandu - tout au moins dans les régions oïliques (Ball 1997). Les travaux de Bulot (1999) sur Rouen démontrent une perception d'un accent Rive-Gauche (populaire, localisé) qui se distingue d'un accent Rive-Droite (classe moyenne, moins localisable) mais qui n'est que partiellement confirmée par les études du comportement (Hall 2008).

\subsection{Une «hiérarchie» des accents et l'insécurité linguistique}

Les études de perception des accents permettent de dresser un tableau assez clair. L'accent parisien est largement reconnu comme le plus correct (Kuiper 2005), et souvent comme le plus agréable à l'oreille - résultat largement confirmé par les travaux consacrés aux français de Belgique et de Suisse (Francard 1993, Singy 1996). En France, on peut noter une seule exception partielle, celle des accents méridionaux, et surtout celui de Provence, largement perçu comme plus agréable que l'accent parisien, et non seulement par des personnes originaires du sud (e.g. Taylor 1996, Blanchet \& Armstrong 2006). Quant aux accents des autres régions, les commentaires valorisants sont peu nombreux. Il n'est guère surprenant que l'accent le plus souvent évoqué par les sujets de Kuiper autre que le leur était celui du Nord-Pas-de-Calais. Le poids de ces représentations négatives contribue à un sentiment d'insécurité linguistique bien documenté (e.g. Lefebvre 1991, Landrecies 2001, Eloy et al. 2003). Mais le sentiment d'insécurité est bien plus largement répandu. L'étude de Caitucoli et al. (2003) en Normandie confirme la dichotomie classique entre le français acquis au sein de la famille et celui que le système éducatif est appelé à enseigner. Des études récentes basées sur un modèle diglossique (e.g. 
Massot et Rowlett 2013, Zribi-Hertz 2013) en distinguant le français «démotique» ou «dialectal» ${ }^{1}$ (Variété $\mathrm{B}$ dans un système soi-disant diglossique) servent à renforcer l'idéologie linguistique du «standard» (Variété A), qui sous-tend ces sentiments d'infériorité et d'insécurité linguistique vis-à-vis le français standard, peu importe l'origine géographique des locuteurs. ${ }^{2}$

\section{L'évolution de la norme et des écarts à cette norme à la lumière des études de comportement}

\subsection{Le système vocalique du français supralocal}
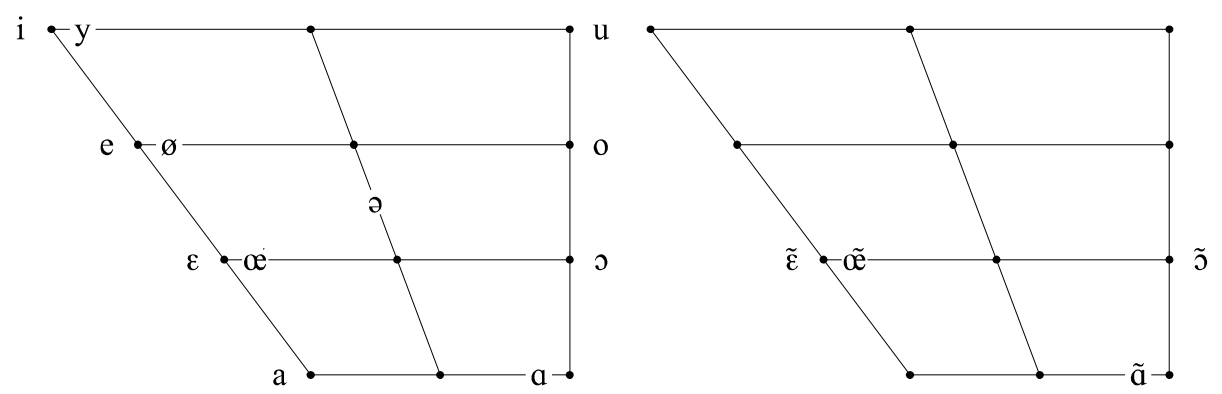

Figure 2: L’inventaire traditionnel des voyelles du français standard (Lyche 2010: 146)

La Figure 2 montre l'inventaire traditionnel des voyelles du français standard, ce qui semble correspondre à la variété cible de la plupart des témoins non-méridionaux ou mobiles de Martinet, ou à la «norme prescriptive» ou «surnorme» de Müller (1985). Une minorité non négligeable des sujets de Martinet, ceux qui avaient grandi dans l'est de la France ou en Bourgogne, croyait se différencier de cette norme par le maintien des oppositions de longueur, (par ex., si [si] scie [si:]) - des contrastes en fort recul depuis le milieu du $20^{\mathrm{e}}$ siècle. Certains témoins, surtout ceux qui étaient originaires du Nord, ne différenciaient pas $/ \mathrm{a} / \sim / \mathrm{a} /{ }^{3}$ et un nombre bien plus important (Paris, Centre, Bourgogne, Nord, Normandie, Ouest) disaient ne pas distinguer

1 Ce terme correspond plus ou moins à français non-standard, informel, parlé.

2 Même si cette «séduction du binaire» est plus que discutable sur le plan de l'analyse linguistique (Gadet et Tyne 2012), elle semble correspondre à une représentation confirmée par des études de perception dans de nombreuses régions francophones.

3 Les habitants du Nord-Pas-de-Calais emploient un $a$ antérieur et un $a$ postérieur, mais la différence est phonétique plutôt que phonémique, et fortement indexée sur le plan social (par ex., Pooley 2004, Hornsby 2006). 


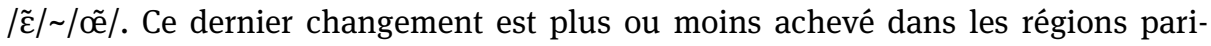
sienne (e.g. Hansen 1998) et lilloise (Pooley 2004), sauf pour l'article indéfini (Østby 2007). Quant aux voyelles moyennes, elles sont de plus en plus réalisées suivant la loi de position. Ceci dit, la charge fonctionnelle de l'opposition $/ \varnothing / \sim / œ /$ est très limitée, ce qui laisse plus de place à des variations phonétiques (e.g. [pør] peur ou [jøn] jeune), parfois présentées comme variantes régionales. Dans les cas où $/ \mathrm{e} / \sim / \varepsilon /$ peuvent faire contraste en syllabe ouverte, et $/ \mathrm{o} / \mathrm{J} / \mathrm{J}$ en syllabe entravée, de plus en plus de locuteurs optent pour une voyelle intermédiaire surtout en syllabe non accentuée. En syllabe accentuée, l'opposition $/ \mathrm{e} / \sim / \varepsilon /$ semble mieux se maintenir pour les désinences grammaticales que pour les noms. L'opposition /o/ / / en syllabe entravée (e.g. Pol [pol], pôle [pol]) reste la plus solide des oppositions entre voyelles moyennes malgré son rendement fonctionnel relativement faible et le fait que sa neutralisation soit considérée comme un trait des variétés du nord et du sud (Carton 1987). La plupart de ces changements que nous avons répertoriés peuvent être classés en termes laboviens comme «changes from below» (Labov 1994), c'est-à-dire des variantes considérées autrefois comme vernaculaires, qui deviennent de plus en plus acceptables. De tels usages sont actuellement admis par certains chercheurs (e.g. Lyche 2010) comme faisant partie du français de référence, alors que d'autres les considèrent comme «démotiques» (Massot 2010) ou «dialectaux» (Zribi-Hertz 2013) par opposition au français standard. Comme il s'agit d'une variété employée majoritairement et sans stigmatisation dans les anciennes régions non-méridionales (Pooley 2006), et qui semble se répandre dans les régions méridionales (Pooley 2007), nous préférons le terme «français supralocal» (Figure 3), et ce sont les écarts diatopiques à cette norme d'usage qu'on peut qualifier de traits régionaux.
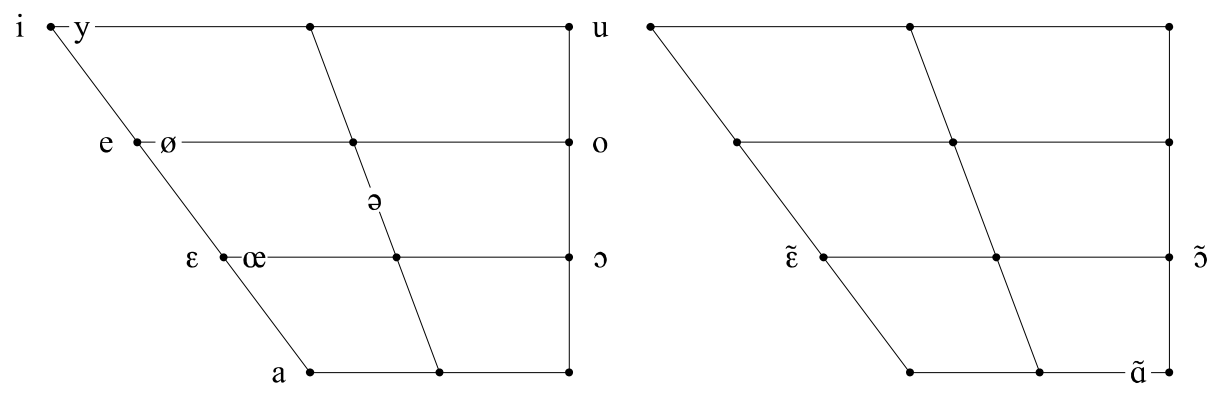

Figure 3: L’inventaire des voyelles du français supralocal

\subsection{Des traits consonantiques supralocaux}

$\mathrm{Au}$ cours de la deuxième moitié du $20^{\mathrm{e}}$ siècle, certains traits consonantiques, souvent mentionnés dans les descriptions des variétés régionales et du français popu- 
laire, sont désormais reconnus comme faisant partie des registres informels du français standard. Le premier est la palatisation des occlusives (e.g. tiens [ $\left.\mathrm{t}^{\mathrm{j} i \tilde{\varepsilon}}\right]$, qui $\left[\mathrm{k}^{\mathrm{j}} \mathrm{i}\right]$ ) phénomène observé dans de nombreux endroits (voir §5.1). Le deuxième est la chute des liquides post-obstruantes (e.g. autre [ot], table [tab]), signalé comme un trait de français non-standard, y compris les variétés régionales plutôt stigmatisé (Detey et al. 2010). Les sujets rennais d'Armstrong et Boughton (1998; 2009) semblent s'être saisis de ce trait pour identifier les personnes des classes populaires, si on croit, comme les auteurs, que leurs témoins évoquaient le phénomène en question par des commentaires tels que: «il bouffe les mots»; «elle mange des lettres»; «il finit pas ses mots». Malgré ces perceptions, les études de comportement montrent bien que les locuteurs des catégories socio-professionnelles supérieures élident assez fréquemment les liquides finales (Østby 2007).

\subsection{L’émergence des variétés régionales dédialectalisées}

L’analyse des corpus de picard et de français enregistrés dans la région lilloise permet d'établir une chronologue approximative d'après les dates de naissance des locuteurs de l'abandon des formes picardes (ou plutôt des formes perçues comme picardes, e.g. une variante avancée de $a$ devant $r$, ou l'absence de $l$ mouillé), et l'adoption systématique de formes perçues comme françaises par les générations qui ont suivi (Tableau 1).

Tableau 1: Chronologie de la perte progressive des traits picards dans les variétés régionales de la région lilloise pratiquées par des locuteurs nés entre 1850 et 1990

\begin{tabular}{|c|c|}
\hline Corpus et années de naissance & Emploi des formes picardes \\
\hline Viez (1910) sujets nés 1850-1860 & Quasi-systématique \\
\hline Carton (1972) sujets nés $1874-1891$ & Variable \\
\hline Carton (1972) sujets nés 1892-1896 & Variable \\
\hline $\begin{array}{l}\text { Carton (1972) un sujets né en } 1895 \text { et les sujets roubai- } \\
\text { siens nés circa } 1910 \text { enregistrés pour Carton et al. (1983) }\end{array}$ & Variable \\
\hline Lefebvre (1991), Pooley (1996) sujets nés avant 1938 & Marginal \\
\hline Lefebvre (1991), Pooley (1996) sujets nés 1939-1952 & Marginal \\
\hline Lefebvre (1991), Pooley (1996) sujets nés 1953-1965 & Plus que marginal \\
\hline Pooley (2004) sujets nés circa 1980 & Rarissime \\
\hline Pooley (2009) sujets nés circa 1990 & Rarissime \\
\hline
\end{tabular}

Même Viez (1910), qui avait pour objectif de décrire le dialecte le plus authentique de Roubaix, montre des signes de francisation. L'industrialisation, l'urbanisation et l'immigration massive de Belges francophones (Pooley 2006b) qui avaient progressé rapidement et de façon significative du vivant de ses principaux témoins, ont coïncidé avec l'émergence de variétés dépicardisées, en partie sous l'effet de la koi- 
néisation et du nivellement provoqué par les échanges entre picardophones, francophones et néerlandophones. Les données recueillies par Carton montrent certes une dépicardisation progressive (indiquée par les sous-divisions par date de naissance), mais les parlers en question demeurent néanmoins nettement plus dialectaux que ceux des témoins qui utilisent le plus de traits picards dans les enregistrements de Pooley (1996). Il ne s'agit pas non simplement du nombre de traits mais aussi de leur fréquence. Même pour les locuteurs nés avant 1938, les traits picards s'emploient à très faible fréquence en comparaison des traits du français régional. Certes, il faut tenir compte des différences diatopiques. La variété la plus différenciée du français se parlait à Gondecourt, village qui se trouve à une dizaine de kilomètres de Lille, mais qui avait plus ou moins complètement échappé à la première vague d'industrialisation (Pouchain 1998). Les enquêtes réalisées dans des localités plus éloignées de Lille - Avion (Hornsby 2006) et le Vimeu (Villeneuve 2011) - ont documenté des pratiques moins dépicardisées que celles observées par Pooley parmi les générations plus âgées, mais un abandon quasi-total chez les plus jeunes.

\subsection{Le nivellement de la dimension diatopique}

Les résultats des études de perception mentionnées au-dessus montrent la difficulté qu'éprouvent les francophones de localiser les locuteurs des régions non-méridionales de façon précise, surtout s'ils sont nés depuis 1970. Tout porte à croire que les habitants des régions concernées n'emploient plus les traits caractéristiques qui les distinguaient au cours de la première moitié du $20^{\mathrm{e}}$ siècle. Le Tableau 2 fournit un exemple typique qui donne lieu de croire que c'étaient les générations nées après la deuxième guerre qui ont abandonné presque totalement les variantes caractéristiques du français local d'origine dialectale encore employées par des Domfrontais (Basse-Normandie) nés entre les deux guerres, en faveur de formes plus largement répandues. Même cet emploi marginal n'est pas suivi par les locuteurs nés dans les années 1980.

On pourrait faire des commentaires similaires sur l'est de la France. Les travaux de Rittaud-Hutinet (1991a; 2001) sur Besançon brossent un tableau d'une variété nettement démarquée de l'usage surpralocal actuel qui était employée par toutes les catégories socio-économiques au moment de l'enquête (circa 1965-68). ${ }^{4}$ À Dijon, Tifrit (2003) indique que certaines oppositions de longueur vocalique persistent parmi des témoins des classes moyennes, nés dans les années 1970 en style lecture, mais becaucoup moins que dans le discours d'une ancienne institutrice dijonnaise née en 1915, dont l'usage se rapprochent des Bisontins enregistrés par Rittaud-Hutinet.

4 Les témoins adultes étaient nés entre 1912 et 1947. 
Tableau 2: L'emploi de quatre variantes régionales par trois générations à Domfront (BasseNormandie, Girard et Lyche 2003)

\begin{tabular}{llll}
\hline & Nés 1920-1933 & Nés 1953-1958 & Nés 1982-1986 \\
\hline $\begin{array}{l}\text { Variantes mi-fermées des } \\
\text { voyelles }\end{array}$ & emploi fréquent & $\begin{array}{l}\text { emploi marginal } \\
\text { emploi fréquent de la } \\
\text { loi de position }\end{array}$ & $\begin{array}{l}\text { loi de position } \\
\text { uniquement }\end{array}$ \\
$\begin{array}{l}\text { Diphtongues } \\
\begin{array}{l}\text { Longueur vocalique } \\
\text { phonémique } \\
\text { rr/apical }\end{array}\end{array}$ & $\begin{array}{l}\text { emploi fréquent } \\
\text { emploi fréquent }\end{array}$ & $\begin{array}{l}\text { emploi marginal } \\
\text { n'emploient pas } \\
\text { n'emploient pas }\end{array}$ & n'emploient pas \\
\hline
\end{tabular}

Les données sur la partie septentrionale des régions d'Oc (Auvergne) semblent indiquer que les habitants ne se distinguent plus de manière facilement audible de leurs compatriotes des régions oïliques. De plus, l'étude de Potte (1977) montre à la fois l'abandon du dialecte local de Glaine-Montaigut (Puy-de-Dôme) et des variantes locales par des personnes nées après 1945. Cela correspond aux changements dans la vie économique de la commune; la grande majorité des anciens avaient travaillé dans le secteur agricole, alors que les plus jeunes cherchaient des emplois en ville.

Si les données perceptuelles sur le français de Bretagne sont relativement abondantes (e.g. Hoare 2004), les études de comportement sont rares. Le travail de Guézennec (2003) sur l'île-de-Sein ne mentionne aucune variable qui manifeste l'influence du breton, ou qui ne soit pas courante dans d'autres régions. Les travaux d'Armstrong et de Boughton sur la Lorraine (Nancy et Dieuze) ont relevé si peu de traits régionaux, qu'ils donnent une plausibilité à l'hypothèse du nivellement quasitotale des marques diatopiques dans les anciennes régions oïliques. Par contre, en Alsace, Pipe (2010) montre que certaines variantes alsaciennes (e.g. le /h/ initial), s'emploient non seulement dans les zones rurales, mais encore à Strasbourg, et cela parmi la plupart des catégories sociales (même si l'analyse en temps apparent suggère fortement qu'ils sont en recul).

\subsection{Le a postérieur - une variante vernaculaire supralocale dans le nord et l'ouest}

Plusieurs enquêtes réalisées dans diverses régions ont permis de documenter la vitalité des $a$ postérieurs surtout en syllabe ouverte finale (e.g. [sa] plutôt que [sa]). Cette variante a été documentée chez des personnes jeunes dans le Nord-Pas-deCalais (Pooley 2004, Hornsby 2006), en Normandie (Girard et Lyche 2003, Hall 2008), en Bretagne (Guézennec 2003) et dans le Jura (Arnaud 2006). Qu'un tel trait soit partagé par plusieurs variétés régionales n'infirme en rien sa valeur identitaire 
pour les usagers d'une variété particulière. ${ }^{5}$ À Saint-Claude, l'emploi de cette variante correspond de façon significative parmi les hommes à un engagement dans la communauté (Arnaud 2006), et dans la métropole lilloise à un indice de «loyauté régionale» parmi des adolescents de diverses origines ethniques (Pooley 2000; 2009). Si ces résultats révèlent des différences significatives, la valeur identitaire n'est décelable qu'au sein de réseaux de contact qui ne sont pas forcément faits pour durer, comme par ex., parmi un groupe d'élèves d'un collège. D'autres enquêtes seraient nécessaires pour montrer si un fort usage du a postérieur correspond à une identité franco-française et masculine, mais ces exemples suffisent à démontrer que divers groupes dans des lieux et des situations différents, peuvent s'approprier un même trait linguistique pour indexer une identité particulière qui ne correspond pas forcément à sa valeur historique.

\section{Des différences régionales émergentes?}

\subsection{Le «français des banlieues»}

Si des variantes régionales classiques peuvent être récupérées par différents groupes dans certaines circonstances, des variantes vernaculaires peuvent aussi prendre une valeur nouvelle et divergente dans des lieux différents. C'est bien le cas de la palatalisation - variante considérée comme fortement vernaculaire dans le Nord-Picardie (Eloy et al. 2003), dans une banlieue parisienne (Jamin 2005), à Grenoble (Trimaille 2003) et à Marseille (Binisti et Gasquet-Cyrus 2003, Jamin et al. 2006). Vu l'importance de la palatisation dans leurs enquêtes respectives, Jamin et al. (2006) ont formulé l'hypothèse de «la convergence dans la divergence». Certes, il faut reconnaître dans ces trois études la forte présence de cette variante audible pour les locuteurs ordinaires dans le parler des jeunes d'origine maghrébine, mais cela n'empêche pas qu'elle puisse prendre aussi une valeur différente sur le plan local. À la Courneuve (Jamin 2005), la palatalisation pourrait être considérée en termes laboviens comme un «marqeur» (Labov 1994) ayant à la fois une valeur stylistique et sociale, car elle est employée plus en conversation spontanée (par opposition au style lecture) par les jeunes de 15 à 25 ans que les adultes, les garçons plus que les filles, et les jeunes d'origine maghrébine plus que ceux issus d'une autre ethnicité, y compris ceux de famille franco-française (Armstrong et Jamin 2002). À Grenoble, les jeunes d'origine maghrébine observés par Trimaille (2003) l'emploient dans la grande majorité des contextes phonologiques aussi bien que dans la plupart des situations observées à un taux de fréquence peu différent (un «indicateur» selon

5 D’autant plus que des différences phonétiques ont été relevées (voir Girard et Lyche 2003). 
Labov 1994). À Marseille, les travaux de Gasquet-Cyrus sont basés uniquement sur des perceptions, et ses résultats indiquent que la palatalisation est associée aux quartiers nord et aux jeunes d'origine maghrébine. Il s'agit plutôt d'un «stéréotype» (Labov 1994: 78), qui ne correspond peut-être plus au comportement actuel.

Les banlieues des grandes villes sont certainement une source féconde de néologismes lexicaux empruntant des termes à plusieurs langues d'immigration. Les locuteurs font preuve d'une grande inventité dans l'usage qu'ils font d'éléments français, supralocaux, régionaux, argotiques, vernaculaires. Il est, par contre, plus problématique de signaler des innovations sur le plan phonologique. Comparons par exemple les traits marqués employés par un témoin (chauffeur-livreur, 22 ans) de Carton et al. (1983) enregistré dans la banlieue nord de Paris (Drancy) dans les années 1970, et des locuteurs multi-ethniques du corpus recueilli par Jamin (2005) à La Courneuve dans les années 1990.

Les traits relevés surtout dans les données des années 1970 sont, soit en fort recul $(2 \mathrm{~b}, 3,6)$, soit se propagent parmi l'ensemble des locuteurs $(1,4)$. Les trois variantes qui sont, nous semble-t-il, les plus fortement indexées sur le plan social et qui figurent largement dans les deux corpus sont les 7, 8 et 9. La distribution lexicale du $a$ postérieur (7) diffère considérablement d'un corpus parisien à l'autre, et on peut constater des divergences avec la Normandie (Hall 2008) et le Nord-Pas-deCalais (Pooley 2004, Hornsby 2006). L'usage de la palatalisation (8), longtemps stigmatisée, commence à se généraliser. Que la réalisation pharyngalisée de /r/ (9) soit un trait ethnique est certainement plausible, car cette variante s'entend bien plus fréquemment chez les jeunes d'origine maghrébine que d'autres adolescents dans différentes régions (Pickles 2001). Il est plausible que deux des trois variantes documentées uniquement dans les années 1990 (traits 10 et 12 du Tableau 3) soient en train de devenir de plus en plus acceptables dans ce que nous avons appelé la variété supralocale. Par contre, le trait 11, la réalisation fermée de $/ \varepsilon /$ est une variante vernaculaire largement répandue dans d'autres régions (par ex., Nord-Pas-de-Calais). 
Tableau 3: Comparaison des traits du vernaculaire parisien dans les années 1970 et 1990 (Carton et al. 1983: 84, Armstrong et Jamin 2002: 132)

\begin{tabular}{|c|c|c|}
\hline Trait & Exemple & Remarque \\
\hline \multicolumn{3}{|l|}{ (a) Années 1970s uniquement } \\
\hline 1. /د/ non accentué antériorisé & joli [zali](ouvert) & [zœli] courant en français \\
\hline ou ouvert & [zœli] (antériorisé) & supralocal \\
\hline 2. /a/ relevé vers [æ] & a) devant $r$ tard [tæR] & S'emploie beaucoup \\
\hline & $\begin{array}{l}\text { b) séquence orthographique } \\
\text { oi par ex., quoi [kwæ] }\end{array}$ & $\begin{array}{l}\text { moins mais se maintient } \\
\text { dans certaines régions }\end{array}$ \\
\hline 3. Postériorisation de/ $/ \tilde{\varepsilon} /$ & 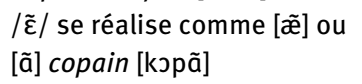 & Devenu marginal \\
\hline $\begin{array}{l}\text { 4. Variante postériorisée et } \\
\text { arrondie de /ã/ }\end{array}$ & argent [æRßว̃] & $\begin{array}{l}\text { Observé en français } \\
\text { supra }\end{array}$ \\
\hline $\begin{array}{l}\text { 5. Affaiblissement des consonnes } \\
\text { intervocaliques }\end{array}$ & avoir [awæR] & $\begin{array}{l}\text { local } \\
\text { Éventuellement causé } \\
\text { par un débit rapide }\end{array}$ \\
\hline $\begin{array}{l}\text { 6. Accent tonique sur la } \\
\text { penultième }\end{array}$ & café ['kafe] & $\begin{array}{l}\text { Devenu marginal mais } \\
\text { encore employé par des } \\
\text { locuteurs âgés }\end{array}$ \\
\hline
\end{tabular}

b) Traits notés dans les deux corpus

7. /a/ d'arrière

8. Palatalisation et/ou affrication des occlusives dentales et vé-

laires

9. /r/ pharyngalisé $\left[\mathrm{b}^{3}\right]$
Années 1970: Fortement postériorisé, paraît dans les items se terminant par $a z$, âtre, âdre: gaz [ga:z] verdâtre [verda:t]

Années 1990: Différence phonétique la table [la tab]; c'est grave [s\& grav] Tiens $[\mathrm{f} \tilde{\mathrm{j}} \tilde{\varepsilon}]$ qui $[\mathrm{k} \mathrm{j}]$

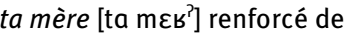
façon variable par un coup de glotte

c) Années 1990 uniquement 10. Relèvement de $/ \mathrm{J} /$ vers $/ \mathrm{o} /$ 11. Relèvement de $/ \varepsilon /$ vers $/ \mathrm{e} /$ devant $/ \mathrm{r} /$

12. Relèvement et allongement de /œ/ vers [ø:] devant $/ r /$ mort [тов] ; police [polis] Marginal

mère [тек] ; vert [рек] Marginal

J'ai peur [рø:s] Commence à

s'entendre dans des

variétés standardisées 


\section{De nouvelles formes de divergence}

\subsection{D'autres cas d'appropriation locale de variantes vernaculaires plus répandues}

Les travaux variationnistes de ce qu'Eckert (2012) appelle les $2^{\mathrm{e}}$ et $3^{\mathrm{e}}$ vagues font appel à des catégories sociales informelles comme des réseaux de contact ou des communautés de pratique. Pour les variétés régionales pratiquées en Europe, plusieurs études ont permis de répandre de la lumière sur les réseaux créés par le milieu professionnel, e.g. Noirmoutier (pêche) (Léonard 2002) ; du sud du Poitou (Auzanneau 1998) et du Puy-de-Dôme (Potte 1977) (agriculture); et du domaine francoprovençal (Kasstan 2015) pour des variétés autochtones, même s'il est parfois délicat de séparer, du point de vue de la distribution sociale, la pratique d'une langue ancestrale et l'emploi de traits dialectaux en français. Dans d'autres cas, il s'agit sans ambiguïté du français, par exemple, l'assourdissement des consonnes finales à Roubaix (Pooley 1994; 2001) et à Mons (Bauvois 2001; 2002). Dans ces deux villes, la variation de type «sociolinguistic gender pattern» (Labov 2001) est/était inversée, car ce sont les femmes travaillant ou ayant travaillé dans certains métiers qui assourdissent plus que leurs collègues masculins.

Les études de Pooley (2000; 2004) dans la métropole lilloise fournissent un autre exemple frappant de l'inversement du modèle classique de la variation sexolectale, celui du o ouvert en syllabe fermée. Parmi une classe de collégiens à Marcqen-Barœul, les filles d'origine étrangère utilisent cette variable de façon radicalement différente des autres : plus de $o$ fermés en lecture, et plus de $o$ ouverts en conversation spontanée. Un corpus enregistré dix ans plus tard (Pooley 2009) confirme l'appropriation ethnique de cette variante parmi des collégiens à Lille-Sud, c'est-àdire que les élèves d'origine maghrébine l'emploient de façon bien plus fréquente que leurs camarades d'origine européenne.

Qu'il nous soit permis également de rappeler l'importance de l'indice de loyauté régionale qui est en corrélation significative avec une valeur élevée de cet indice ${ }^{6}$ pour l'emploi fréquent du $a$ postérieur et du $o$ ouvert en syllabe entravée. Un aspect du travail de Pooley (2009) permet d'analyser à un niveau plus détaillé des «communautés de pratique» (Eckert 2000) parmi les classes de collégiens étudiées. Il s'agit en l'occurrence d'indices d'amitié. On a demandé aux témoins de nommer leurs trois meilleurs amis à l'intérieur et à l'extérieur du collège. Les réponses fournies permettent dans une certaine mesure d'évaluer premièrement, le degré de contact interethnique dans les relations personnelles de chacun, et deuxièmement, la

6 Une valeur maximale de 3 indique qu'un témoin a une attitude positive envers la région et ses habitants et déclare son intention de vivre dans la région en préférence à d'autres possibilités au cours de sa vie d'adulte. 
popularité de chaque élève au sein de sa classe. Grâce à cette démarche, deux indices d'amitié ont été créés: premièrement un indice d'amitié interethnique au collège (IAIC), et deuxièmement, un indice d'amitié interethnique en dehors du collège (IAID), basé simplement sur les prénoms mentionnés qui ne correspondent pas à l'ethnicité du témoin. Pour le troisième indice, il s'agit de l'indice de popularité en classe (IPC) basé sur le nombre de fois qu'un individu a été cité comme ami/e par ses camarades. Les indices IAIC et IAID s'avèrent être en corrélation négative avec l'emploi fréquent du $a$ postérieur et du o ouvert en syllabe entravée, ce qui semble confirmer l'hypothèse de l'ethnication de ces variantes sur le plan local. Par contre, les franco-français qui ont une valeur positive pour les deux indices pratiquent davantage le «crossing» ${ }^{7}$ (Rampton 2005, Pooley et Mostefai-Hampshire, 2012). Mais ce sont les témoins ayant une valeur élevée de l'indice IPC qui utilisent le plus les deux variantes vernaculaires, ce qui semble indiquer une plus forte implication dans la sous-culture adolescente.

\subsection{Des différences indécelables à l'oreille}

Si tous ces cas de variation ne se laissent déceler que par une analyse détaillée de facteurs microcosmiques, ils correspondent néanmoins à des réalités sociales, alors que d'autres différences ne se révèlent que grâce à des analyses statistiques ou comparaison inter-régionales.

Ayant remarqué que la fréquence de la chute des liquides post-occlusives différait de façon considérable chez ses sujets rennais et nancéiens, Boughton (2013) évoque la possibilité de l'émergence de nouvelles formes de variation régionales (Tableau 4).

$\mathrm{Vu}$ la réalisation généralement plus fréquente du schwa dans les variétés méridionales, il n'est guère surprenant que les Rodésiens ou Ruthénois suppriment les liquides finales bien moins souvent que leurs homologues non méridionaux, et même les Aveyronnais installés dans la région parisienne. Il aurait pu être tentant de postuler une différence entre l'est et l'ouest, vu la similitude des résultats pour Strasbourg (Pipe 2010) et Nancy et l'écart d'environ 20\% avec Rennes, mais cette hypothèse est infirmée par les résultats du Vimeu (Villeneuve 2009) et de Dieuze en Lorraine (Armstrong 1993). Dans les régions non-méridionales, les autres grandes catégories sociales sont certainement plus importantes. En général, les femmes prononcent les liquides plus que les hommes (Roubaix, Rennes, Nancy), mais Pipe (2010) observe une situation inversée à Strasbourg. On note plus de régularité dans

7 L'emploi d'une langue acquise informellement qui ne correspond pas à l'ethnicité du locuteur, surtout l'emploi de mots et d'expressions arabes par les jeunes d'origine franco-française. 
le comportement des locuteurs d'âge et de classe sociale différent. Les locuteurs plus âgés et les classes populaires élident plus que les jeunes (Vimeu, Nancy, Rennes) et les classes populaires plus que les classes moyennes (Nancy, Rennes). $\mathrm{Vu}$ la difficulté éprouvée par les témoins nancéiens et rennais à localiser leurs homologues de l'autre ville même sur la base d'un extrait relativement long, sans oublier le fait que tous les locuteurs non-méridionaux l'emploient à un taux de fréquence non négliegeable, il nous paraît néanmoins indéniable que ce trait ne permet pas de les localiser (voir §3). Se peut-il qu'on ait affaire à une variable dit «hyperstyle» ${ }^{8}$ (Armstrong 2013) ? Probablement pas, car la variation correlée aux classes sociales des locuteurs correspond plutôt au pattern typique d'un marqueur où la variante vernaculaire apparaît bien plus en conversation qu'en style lecture (Tableau 5).

Tableau 4: Taux de fréquence de la chute des liquides post-occlusives en final du mot dans dix régions (d'après Boughton 2013)

\begin{tabular}{ll}
\hline & Classes moyennes \\
\hline Montréal (Kemp et al. 1980) & $72 \%$ \\
Nancy (Boughton 2005) & $47 \%$ \\
Rennes (Boughton 2005) & $67 \%$ \\
Strasbourg (Pipe 2010) & $49 \%$ \\
\hline & Classes populaires \\
\hline Avion (Hornsby 2006) & $80 \%$ \\
Banlieue de Paris (Laks 1977) & $77 \%$ \\
Roubaix (Pooley 1996) & $69 \%$ \\
\hline & Classes non précisées \\
\hline Dieuze (Armstrong 1993) & $70 \%$ \\
Vimeu (Villeneuve 2009) & $62 \%$ \\
Rodez (Pustka 2007) & $6 \%$ \\
Aveyronnais à Paris (Pustka 2007) & $22 \%$ \\
\hline
\end{tabular}

Tableau 5: La chute des liquides post-occlusives en finale du mot. Variation stylistique. (Boughton 2013)

\begin{tabular}{llll}
\hline & Liste de mots & Lecture de texte & Entretien \\
\hline Nancy & $6 \%$ & $29 \%$ & $47 \%$ \\
Rennes & $10 \%$ & $49 \%$ & $67 \%$ \\
\hline
\end{tabular}

8 Une variable qui manifeste plus de différences sur le plan stylistique que sur le plan social. 
Les analyses instrumentales réalisées sous l'égide du Projet du Français Contemporain ont permis de déceler des différences qui avaient échappé à l'oreille des locuteurs ordinaires, sans parler d'un bon nombre de spécialistes. Par exemple, Lyche (2011) montre que les locuteurs suisses prononcent environ 10\% de plus de schwas que les locuteurs des régions oïliques de France, alors que les études classiques (e.g. Walter 1982) affirment que les Romands suivent de près les pratiques nonméridionales de l'Hexagone. Les résultats présentés par Durand et Lyche (2008) sur les liaisons variables suggèrent que les non-méridionaux les réalisent moins souvent que les méridionaux, mais les auteurs nous mettent en garde contre la formulation trop rapide d'une hypothèse.

\subsection{Le schwa prépausal: une innovation parisienne?}

Dans deux études basées sur des corpus enregistrés à Paris, Hansen (1998), Hansen et Hansen (2003) analysent un phénomène qu'elles appellent le schwa prépausal (e.g. c'est Pierre-euh, bonjour-euh). Au niveau pragmatique, cette voyelle finale a la fonction de mettre en exergue un élément important du discours. Au niveau phonologique elle sert à marquer la fin d'un groupe, d'autant plus que, contrairement aux /ə/ finals des variétés méridionales, elle est accompagnée d'une intonation descendante. Il s'agit peut-être d'un phénomène phonotactique qui émerge naturellement du système phonologique, et qui, par la suite a acquis une distribution sociolinguistique. Ce sont les jeunes femmes qui sont à l'initiative dans l'adoption de ce trait qui, semble-t-il, est plus employé dans les styles relativement formels. Même s’il s'agit d'une innovation qui va durer, le schwa prépausal était encore une variante minoritaire (utilisée dans $25 \%$ des cas possibles) dans le $2^{\text {ième }}$ corpus (circa 1993).

\section{Conclusion - vers une nouvelle carte de la variation régionale?}

Si les variétés régionales du français peuvent être caractérisées par des écarts à la norme, il faut d'abord reconnaître que cette norme a considérablement évolué depuis le milieu du $20^{\mathrm{e}}$ siècle. Il ne s'agit plus d'une norme prescriptive (Müller 1985) (bien que celle-ci exerce encore une influence non négligeable dans l'Imaginaire linguistique des francophones), mais d'une norme d'usage employée par une majorité de la population de l'Hexagone (Figure 4), d'où l'intérêt du terme français supralocal (ou même un français «passe-partout») survient, qui ne permet pas de déceler les origines géographiques des locuteurs, tout au moins en France. 


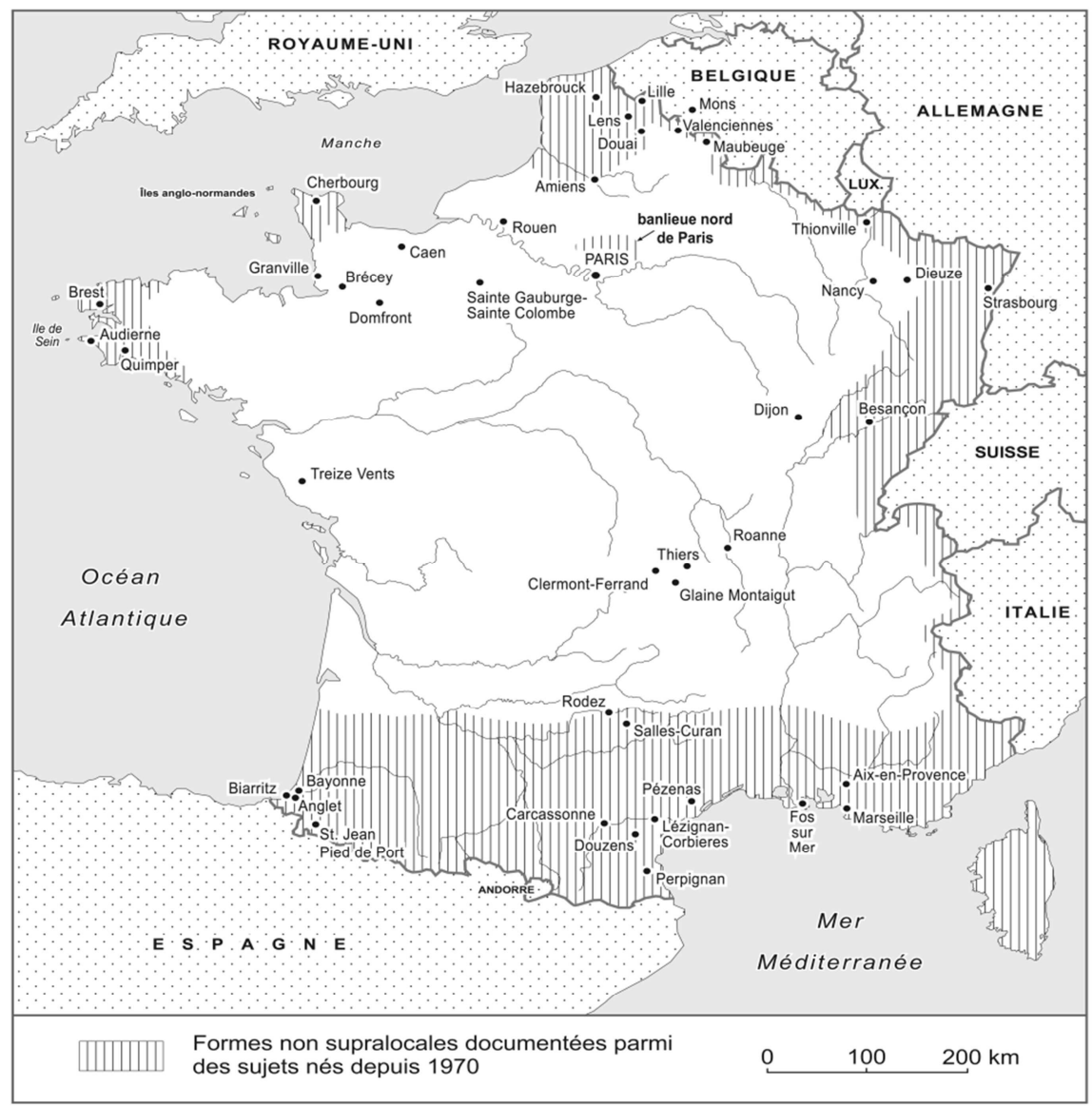

Figure 4: Régions où des locuteurs nés depuis 1970 emploient des variantes non supralocales de façon variable

Nous avons vu que les traits proprement dialectaux qui permettaient d'identifier ce qu'on appelle parfois les français régionaux, ne s'entendent quasiment pas dans la bouche de locuteurs nés depuis 1970 et toutes les variantes françaises considérées comme marques régionales sont en fort recul. Mais certaines de ces variantes ( $a$ postérieur ; $o$ fermé en syllabe entravée) ont pris une valeur identitaire dans des conditions particulières - un phénomène documenté ailleurs dans des travaux sociolinguistiques (par ex., Moore 2010 en Angleterre). Nous avons souligné par exemple la différenciation parmi les groupes de jeunes multi-ethniques à Lille.

Si les différences diatopiques classiques s'estompent, de nouveaux écarts à la norme à la fois localisables sur le plan géographique et reconnaissables sur le plan social émergent bien plus timidement que les néologismes lexicaux dans les mêmes circonstances, et ces innovations phonétiques ont été observées dans les banlieues 
de plusieurs grandes villes. De ce fait-là, l'hypothèse de la convergence dans la divergence s'avère séduisante mais loin d'être solidement étayée. Au contraire, l'isolement social et géographique d'un grand nombre de ces quartiers et le manque d'échanges directes la rend plutôt peu plausible. Ce qui a été documenté, par contre, est la récupération de variantes vernaculaires (régionales, sociales ou ethniques) pour servir de marqueur identitaire sur le plan local.

Des enquêtes réalisées depuis 1980 ont permis de déceler des différences diatopiques qui n'ont apparemment aucune indexation sociale. Il s'agit de variantes historiquement vernaculaires, mais qui sont en passe de devenir de plus en plus acceptables dans les variétés standardisées (e.g. la chute des liquides en finale). Il semblerait que même si la comparaison des taux de fréquence dans divers lieux révèlent des différences significatives sur le plan statistique, celles-ci n'aient apparemment aucune importance au niveau perceptuel, et donc social.

Si en matière de prononciation, on peut encore parler de variétés régionales $d u$ français, le nombre de régions concernées est bien réduit, car les sous-divisions historiques de la partie septentrionale de la France s'estompent dans la prononciation de locuteurs nés depuis 1970, et la frontière oïlique/nord Occitan est en fluctuation. Il est plausible que Paris reste un cas particulier (Durand 2010), et la capitale est toujours un centre d'innovation, comme le montre le cas du schwa prépausal.

De nouvelles variétés qui sont en partie définissables sur le plan géographique ont vu le jour dans les banlieues. Il faut donc se pencher plus sur la variation dans les quartiers multi-ethniques, un champ d'enquête toujours très peu exploité, comme Jones \& Hornsby (2013) ont souligné. Cependent, l'émergence de ces variétés dépend davantage de facteurs sociaux que géographiques. Même si certaines variantes (par ex., la palatalisation des occlusives sourdes) ont été observées dans la prononciation d'adolescents dans différentes villes très éloignées les unes des autres, il est difficile d'étayer l'hypothèse de la convergence dans la divergence, car l'indexation sociale de ce phénomène est franchement différente dans toutes les villes étudiées. D’ailleurs, les locuteurs (Paris, Lille, Marseille) maintiennent souvent des traits vernaculaires typiques des variétés régionales classiques.

\section{Références}

Armstrong, N., (2001), Social and stylistic variation in spoken French, Amsterdam, Benjamins. Armstrong, N., (2013), «Hyperstyle variation in French: Yet another exception culturelle ? dans M. C. Jones \& Hornsby, D., (dir.), Language and social structure in urban France, Oxford, Legenda, 81-93.

Armstrong, N. \& Boughton, Z., (1998), «Identification and evaluation in responses to a French accent; some results and issues of methodology, Paroles 5,6, p.27-60.

Armstrong, N., (2009), Perception and production in French dialect levelling, dans K. Beeching, N. Armstrong \& F. Gadet (dir.), 9-24. 
Armstrong, N. \& Jamin, M., (2002), «Le français des banlieues: uniformity and discontinuity in the French of the Hexagon», dans K. Salhi (dir.), French in and out of France. Language policies, intercultural antagonisms and dialogue, Oxford, Peter Lang, 107-36.

Amstrong, N. \& Pooley, T., (2010), Social and linguistic change in European French, Basingstoke, Palgrave.

Arnaud, V., (2006), «La dimension variationniste du français en usage à Saint-Claude (Haut-Jura): Une étude acoustique des voyelles orales des "gens d'en haut»», Thèse de doctorat, Université Laval.

Auzanneau, M., (1998), La parole vive du Poitou. Une étude sociolinguistique en milieu rural poitevin, avec application aux marchés, Paris, L'Harmattan.

Ball, R., (1997), The French-speaking world, Londres, Routledge.

Bauche, H., (1946), Le langage populaire, Paris: Payot.

Binisti, N., \& Gasquet-Cyrus, M., (2003), «Les accents de Marseille», Cahiers du Français Contemporain, 8, 107-30.

Blanchet, P., \& Armstrong, N., (2006), «The sociolinguistics of 'contemporary dialects of French' in France today: an overview of recent contributions on the dialectalisation of Standard French», Journal of French Language Studies, 16, 3, 251-275.

Boughton, Z., (2005), «Accent levelling and accent localisation in northern French: Comparing Nancy and Rennes», Journal of French Language Studies, 15, 3, 235-256.

Boughton, Z., (2006), «When perception isn't reality: Accent identification and perceptual dialectology in French », Journal of French Language Studies, 16, 3, 277-304.

Boughton, Z., (2013), «Locating variation in French: Geolinguistic patterns, levelling, and the "French exception"», dans M. C. Jones \& Hornsby, D., (dir.), Language and social structure in urban France, Oxford, Legenda, 119-132.

Brun, A., (1946), Parlers régionaux, France dialectale et unité française, Paris, Didier.

Bulot, T., (1999), «La production de l'espace urbain à Rouen: mise en mots de la ville urbanisée», dans T. Bulot \& N. Tsekos, Langue urbaine et identité (Langue et urbanisation linguistique à Rouen, Venise, Berlin, Athènes et Mons). Paris, L'Harmattan, 39-70.

Caitucoli, C., Delamotte-Legrand, R., \& Leconte, F., (2003), «L'usage du langage dans l'institution scolaire: Variation et perception de la variation», Cahiers du Français Contemporain, 8, 19-34.

Carton, F., (1987), "Les accents régionaux», dans G. Vermes \& J. Boutet (dir.) France, pays multilingue, Paris, L'Harmattan, Vol II, 29-49.

Carton, F., (2001), «Quelques évolutions récentes dans la prononciation du français», dans M.-A. Hintze, T. Pooley \& A. Judge (dir.), French accents: Phonological and sociolinguistic perspectives, Londres, AFLS/CiLT., 7-23.

Carton, F. \& Lebègue, M. (1989). Atlas linguistique et ethnographique picard, Paris, Editions CNRS.

Carton, F., \& Rossi, M., Autesserre, D., \& P. Léon, (1983), Les accents des Français, Hachette, Paris.

Cerquiglini, B., (1999), «Les langues de la France», Rapport au Ministre de l'Éducation Nationale, de la Recherche et de la Technologie, et à la Ministre de la Culture et de la Communication.

Dauzat, A., (1906), Essai de méthodologie dans le domaine des langues et des patois romans, Paris, Champion.

Detey, S., Durand, J., Laks, B. \& Lyche, C., (dir.), (2011), Les variétés du francais dans l'espace francophone: ressources pour l'enseignement, Paris, Ophrys.

Detey, S. \& Le Gac, D., (2010), «Le français de référence: quels locuteurs», dans S. Detey et al., Paris, Ophrys, p. 167-180.

Durand, J. \& Lyche, C. (2008), «French liaison in the light of corpus data», Journal of French Language Studies, 18, 1, p.33-66.

Eckert, P. (2000), Linguistic Variation as Social Practice, Oxford, Blackwell. 
Eckert, P., (2012), «Three waves of variation study: The emergence of meaning in the study of sociolinguistic variation», Annual Review of Anthropology, 41, p.87-100.

Eloy, J.-M., Blot, D., Carcassone, M. \& Landrecies, J., (2003), Français, picard, immigrations. Une enquête épilinguistique, Paris, L'Harmattan.

Flutre, L.-F. (1977). Du moyen picard au picard moderne, Amiens, Musée de Picardie.

Francard, M., (1993), L'insécurité linguistique, Bruxelles, Duculot.

Gadet, F., (1996), «Variabilité, variation, variété dans le français d'Europe», Journal of French Language Studies, 6, 1, 75-98.

Gadet, F. \& H. Tyne, (2012), «La seduction du binaire», dans T.Pooley \& D. Lagorgette (dir.) On linguistic change in French. Socio-historical approaches, Chambéry, Presses universitaires de Chambéry, 55-68.

Gasquet-Cyrus, M., (2004), «The sociolinguistics of Marseille», International Journal of the Sociology of Language, 169, p.107-123.

Gasquet-Cyrus, M., (2009), «Territorialisation, stigmatisation et diffusion: l'accent 'quartiers nord' à Marseille», dans T. Bulot (dir.) Formes et normes sociolinguistiques: ségrégations et discriminations urbaines. Paris, L'Harmattan, 209-222.

Gilliéron, J. \& E. Edmont. (1902), Atlas linguistique de la France. Notice, servant à l'intelligence des cartes. Paris, Champion.

Girard, F., \& Lyche, C., (2003), «La phonologie du français contemporain dans le Domfrontais: un français en évolution», Tribune Internationale des Langues Vivantes, 33, 166-73.

Guézennec, N., (2003), «Variations phonétiques: entre identité et interactions sociales. Cas de l'île de Sein (Finistère-Sud)», Tribune Internationale des Langues Vivantes, 33, 151-158.

Hambye, P., (2005), La prononciation du français contemporain en Belgique. Variation, normes et identités. Thèse de doctorat, Université catholique de Louvain.

Hansen, A.-B., (1998), Les voyelles nasales du français parisien moderne, Copenhague, Museum Tusculanum.

Hansen, A.-B. \& Hansen, M.-B., (2003), «Le [ə] prépausal et l'interaction», dans A. Hansen \& M.-B. Hansen (dir.) Structures linguistiques et interactionnelles dans le français parlé, Copenhagen, Museum Tusculanum, p. 89-109.

Hansen, A.-B. \& Juillard, C., (2011), «La phonologie parisienne à trente ans d'intervalle - Les voyelles à double timbre», Journal of French Language Studies 21, 3, p.313-359.

Hall, D., (2008), A sociolinguistic study of the regional French of Normandy, Thèse de doctorat, University of Pennsylvania.

Hauchecorne, F. \& Ball, R., (1997), L'accent du Havre: Un exemple de mythe linguistique, Langage et société, 82, 5-26.

Hoare, R., (2004), «Language attitudes and perceptions of identity in Brittany», Teanga (Irish Yearbook of Applied Linguistics), 20, 163-92.

Hornsby, D., (2006), Redefining Regional French: Koinéization and Dialect Levelling in Northern France. Oxford, Legenda.

Jamin, M., (2005), Sociolinguistic variation in the Paris suburbs, Thèse de doctorat, University of Kent.

Jamin, M., Trimaille, C., \& Gasquet-Cyrus, M., (2006), «De la convergence dans la divergence: le cas des quartiers pluriethniques en France», Journal of French Language Studies, 16, 3, 335-56.

Jones, M. C. \& D. C. Hornsby, (2013), Introduction dans M.C. Jones \& Hornsby, D., (dir.) Language and Social Structure in Urban France, Oxford, Legenda, 1-7.

Kasstan, J. R. (2015). Variation and change in Francoprovençal: A study of an emerging linguistic norm. Thèse de doctorat, University of Kent.

Kuiper. L. (2005). «Perception is Reality: Parisian and Provençal perceptions of regional varieties of French», Journal of Sociolinguistics, 9, 1, 28-52. 
Labov, W., (1966), The Social Stratification of English in New York City, Washington, DC : Center for Applied Linguistics.

Labov, W., (1994), Principles of Linguistic Change. Volume 1 : Internal Factors. Oxford: Blackwell.

Labov, W., (2001), Principles of Linguistic Change. Volume 2 : External Factors. Oxford: Blackwell.

Laks, B., (1977), «Contribution empirique à l'analyse socio-différentielle de la chute des /r/ dans les groupes consonantiques finals», Langue française 34, p109-125.

Landrecies, J., (2001), «'C'est laid mais ça me fait rire': Les représentations de l'accent du Nord dans une population de stagiaires de l'IUFM de Lille», dans M.-A. Hintze, T. Pooley \& A. Judge (dir.), French accents: Phonological and sociolinguistic perspectives, Londres, AFLS/CiLT., 196-217.

Lefebvre, A., (1991), Le français de la région lilloise. Paris: Publications de la Sorbonne.

Léonard, J.-L., (2002), «Microcosmic perceptual dialectology and the consequences of extended linguistic awareness. A case study of Noirmoutier Island (France)», dans D. Preston \& D. Long (dir.), Handbook of perceptual dialectology. Vol 2., Amsterdam, John Benjamins, 219-248.

Lodge, R.A., (2004), A sociolinguistic History of Paris, Cambridge, Cambridge University Press.

Lyche, C., (2010), «Le français de référence: éléments de synthèse», dans S. Detey et al., Paris, Ophrys, 143-165.

Martinet, A., (1945), La prononciation du français contemporain, Paris, Droz.

Massot, B., (2010), «Le patron diglossique de variation grammaticale en français», Langue française. 87-106.

Massot, B. \& Rowlett, P., (2013), «Le débat sur la diglossie en France: aspects scientifiques et politiques», Journal of French Language Studies, 23,1, 1-16.

Moore, E., (2010), «Communities of Practice and peripherality», dans C. Lllamas \& D. Watt (dir.) Language and Identities, Edinburgh, Edinburgh University Press: 123-133.

Mougeon, R., (1996), «La recherche sociolinguistique sur le français du Canada» dans J. Erfurt (dir.), De la polyphonie à la symphonie: Méthodes, théories et faits de la recherche pluridisciplinaire sur le français au Canada. Leipzig : Leipziger Univ.-Verl., p.183-206.

Müller, B., (1985), Le français d'aujourd'hui, Paris, Klincksieck.

Østby, K., (2007), «Aspects du français oral: la haute bourgeoisie parisienne», Bulletin PFC, 7, 227-36. Projet Phonologie du Français Contemporain. http://j.mp/PFCBulletin7.

Pedersen, I.-L., (1994), Linguistic variation and composite life modes, dans B. Nordberg (dir.), The sociolinguistics of urbanization: the case of the Nordic countries, Berlin, Walter de Gruyter: 87-115.

Pickles, M., (2001), «La mère de mon père est née à Grenade: Some phonological features of the French of teenagers in Perpignan», dans M.-A. Hintze, T. Pooley \& A. Judge (dir.), French accents: Phonological and sociolinguistic perspectives, Londres, AFLS/CiLT., 128-147.

Pipe, K. (2010), «Phonological variation and change in Alsatian French », Mémoire de maîtrise, University of Exeter.

Pooley, T., (1996), Chtimi: The Urban Vernaculars of Northern France. Clevedon: Multilingual Matters.

Pooley, T., (2000), «The use of regional French by Blancs and Beurs: questions of identity and integration in Lille», Interface, 5, 51-69.

Pooley, T., (2004), Language, dialect and identity in Lille. 2 Vols. Lewiston NY: Edwin Mellen.

Pooley, T., (2006a), «On the geographical spread of Oïl French in France», Journal of French Language Studies, 16, 3, 357-390.

Pooley, T., (2006b), «The linguistic assimilation of Flemish immigrants in Lille» (1800-1914), Journal of French Language Studies, 16, 2, 207-233.

Pooley, T., (2007), «Dialect levelling in southern France», Nottingham French Studies, 46, 2, 40-63. 
Pooley, T., (2009), «The immigrant factor in phonological levelling», dans K. Beeching, N. Armstrong \& F. Gadet (dir.), Sociolinguistic variation in contemporary French. Amsterdam/Philadelphia: Benjamins, 64-77.

Pooley, T. \& Mostefai-Hampshire (2012), «Code-crossing and multilingualism among adolescents in Lille ", Journal of French Language Studies, 22, 3, 371-394.

Potte, J.-C., (1977), «Le français de Glaine-Montaigut (Puy-de-Dôme): Connaissance du milieu et approche phonétique», dans G. Taverdet \& G. Straka (dir.), Les français régionaux, Paris, Klincksieck, 191-198.

Pouchain, P., (1998), Les maîtres du Nord du XIXe siècle à nos jours. Lille: Perrin.

Pustka, E., (2007), Phonologie et variétés en contact. Aveyronnais et Guadeloupéens à Paris. Tübingen : Narr.

Rampton, B., (2005), Crossing. $2^{\text {nd }}$ Ed. Manchester, St Jerome.

Reynaud, A., (1981), Société, espace et justice, inégalités régionales et justice socio-spatiale, Paris, Presses universitaires de France.

Rittaud-Hutinet, C., (1991), «Schémas intonatifs des signes vocaux et français régional: le français de Besançon", Lengas, 30, 145-63.

Rittaud-Hutinet, C., (2001), «Les français de Besançon: Systèmes phonologiques et énonciation», dans M.-A. Hintze, T. Pooley \& A. Judge (dir.), French accents: Phonological and sociolinguistic perspectives, Londres, AFLS/CiLT., 96-127.

Singy, P., (1996), L'image du français en Suisse romande, Paris, L'Harmattan.

Taylor, J., (1996), Sound evidence, Berne, Peter Lang.

Trimaille, C., (2003), «Variation dans les pratiques langagières d'enfants et d'adolescents dans le cadre d'activités promues par un centre socio-culturel, et ailleurs», Cahiers du Français Contemporain, 8, 131-62.

Trimaille, C., (2008), Who's not palatalizing ? Trying to understand the status of palatalized variants in French. 8th Conference of the HDLS, Albuquerque, 6-8 novembre.

Trimaille, C. \& Gasquet-Cyrus, M., (2013), «Sociolinguistic change in the city: Gentrification and its linguistic correlates in Marseille», dans M.C. Jones \& D. Hornsby, (dir.), Language and social structure in urban France, Oxford, Legenda: 190-209.

Trudgill, P., (1974), The Social Differentiation of English in Norwich. Cambridge: Cambridge University Press.

Viez, H., (1910) [1978], Le parler populaire (patois) de Roubaix, Marseille, Lafitte Reprints.

Villeneuve, (2011), A sociolinguistic study of Vimeu French. Thèse de doctorat. Indiana University.

Walter, H., (1982), Enquête phonologique et variétés régionales du français, Paris, Presses universitaires de France.

Watt, D., (2002), "'I don't speak with a Geordie accent, I speak, like, the Northern accent': Contactinduced levelling in the Tyneside vowel system», Journal of Sociolinguistics, 6(1), 44-63.

Williams, A., \& Kerswill, P., (1999), «Dialect levelling: Change and continuity in Milton Keynes, Reading and Hull», dans P. Foulkes \& G. Docherty (dir.), Urban voices, Londres, Arnold, 141-162.

Woehrling, C., \& P. Boula de Mareüil, (2006), Identification d'accents régionaux en français: perception et catégorisation. Revue Parole, 37, 25-65.

Zribi-Hertz, A., (2013), «De la notion de grammaire standard dans une optique diglossique du français», Journal of French Language Studies, 23, 1, 59-85. 